\title{
DIABETES \\ On trial-bariatric surgery for treatment of type 2 diabetes mellitus
}

B ariatric surgery improves glycaemic control in patients with long-standing type 2 diabetes mellitus (T2DM) when compared with medical therapy, show two prospective, randomized trials published in The New England Journal of Medicine.

Bariatric surgery has gained popularity as a successful approach to treat patients with obesity. The results of previous studies have suggested that bariatric surgery could also be helpful as a means to control hyperglycaemia in T2DM. Most of these studies, however, were observational or did not include patients with long-standing T2DM.

The study by Philip Schauer and colleagues was performed at the Cleveland Clinic (Cleveland, USA) and involved a population of 150 patients with T2DM, defined as an $\mathrm{HbA}_{1 \mathrm{c}}$ level $>7.0 \%$. Patients were aged $20-60$ years (mean 49 years) and $66 \%$ were women. The mean duration of T2DM at baseline was $>8$ years. In contrast to other studies involving bariatric surgery, in which only people with severe obesity were included, the patients' BMI at baseline ranged from 27 to $43 \mathrm{~kg} / \mathrm{m}^{2}$. Patients were randomly assigned to receive intensive medical therapy alone or undergo Roux-en-Y gastric bypass surgery or sleeve gastrectomy in addition to intensive medical therapy.

At the 12-month follow-up, 140 patients (93\%) had completed the study. The primary end point, $\mathrm{a} \leq 6.0 \% \mathrm{HbA}_{1 \mathrm{c}}$ level, was reached by $42 \%$ of patients who underwent gastric bypass surgery and $37 \%$ of patients who underwent sleeve gastrectomy, whereas only $12 \%$ of patients who received intensive medical therapy alone reached this target.

The study by Geltrude Mingrone and colleagues included 60 patients with $\geq 5$ years of T2DM duration who were recruited at the Day Hospital of Metabolic Diseases and Diabetology of the Catholic University in Rome, Italy. T2DM was defined as an $\mathrm{HbA}_{1 \mathrm{c}}$ level $\geq 7.5 \%$. Patients,
$53 \%$ of whom were women, were aged $30-60$ years and had a BMI $\geq 35 \mathrm{~kg} / \mathrm{m}^{2}$ at baseline. Patients were randomly assigned to receive conventional medical therapy, to undergo gastric bypass surgery, or to undergo biliopancreatic-diversion surgery.

At the 2-year follow-up, 56 patients had completed the study. A decrease in $\mathrm{HbA}_{1 \mathrm{c}}$ levels and fasting glucose levels was observed in all groups. However, the changes were more pronounced in the two surgery groups, with $95 \%$ of the patients who underwent biliopancreaticdiversion surgery and $75 \%$ of patients who underwent gastric bypass surgery having fasting glucose levels $<5.6 \mathrm{mmol} / \mathrm{l}$ and $\mathrm{HbA}_{1 \mathrm{c}}$ levels in the absence of pharmacologic therapy $<6.5 \%$. These patients were classified as being in clinical remission for T2DM. By contrast, no patients in the medical therapy group were in clinical remission. Importantly, "no correlation was observed between T2DM remission and initial body weight nor between T2DM remission and weight loss," Mingrone points out, "suggesting that BMI should not be used as the sole criterion for selection of patients with T2DM for bariatric surgery".

If weight loss does not explain the observed improvement in insulin sensitivity, what are the mechanisms that underlie this effect of bariatric surgery? Mingrone explains that the normalization of the lipid profile in patients who underwent this procedure, which is associated with an improvement in insulin sensitivity, is likely to have been a consequence of lipid malabsorption.

"The rerouting of nutrients and/or their sudden arrival into the ileum, bypassing the duodenum and the jejunum, could explain the results." However, "the improvement in insulin resistance after biliopancreatic diversion is not correlated with the temporal distance from the operation," says Mingrone. "Other mechanisms of glucose control take place in the long run, such as a drastic

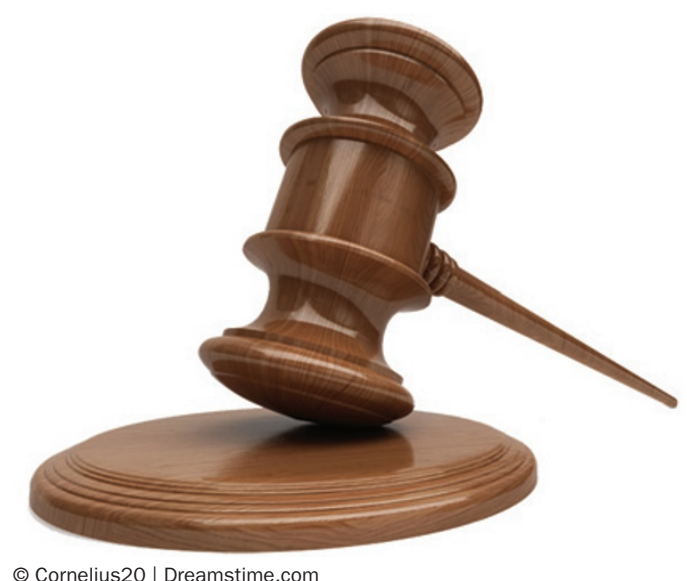

() Cornelius20 | Dreamstime.com

reduction of triglyceride deposition within and inside the skeletal muscle cells as a consequence of lipid malabsorption," she adds. Mingrone and colleagues now plan to study the mechanisms of resolution of T2DM after bariatric surgery at a molecular level.

Both study groups also plan to follow up their patients for several years to assess the durability of glycaemic control and monitor the occurrence and progression of diabetes-related complications.

The results of the two studies are in line with the International Diabetes Federation recommendation that bariatric surgery is an appropriate treatment for patients with a BMI $\geq 35 \mathrm{~kg} / \mathrm{m}^{2}$ and uncontrolled T2DM. However, as Mingrone points out, the new results indicate that criteria other than BMI, such as insulin pancreatic reserve and the degree of insulin resistance, should also be taken into account when selecting patients for bariatric surgery.

Joana Osório

\footnotetext{
Original articles Schauer, P. R. et al. Bariatric surgery versus intensive medical therapy in obese patients with diabetes. N. Engl. J. Med. doi:10.1056/NEJMoa1200225 | Mingrone, G. et al. Bariatric surgery versus conventional medical therapy for type 2 diabetes. N. Engl. J. Med. doi:10.1056/NEJMoa1200111
} 